\title{
CONFERENCE DIARY
}

\section{NOVEMBER 2003}

5-6 Filtration and Separation Symposium, Tokyo, Japan. Details: E-mail: iritani@nuce. nagoya-u.ac.jp

7-8 4th Indian Conference on Computer Applications in Mineral Industry ICCAMI 2003, Bhubaneswar, Orissa, India. Details: E-mail: dir@rrlbhu.res.in, Website: http:// www.rrlbhu.res.in

17-19 5th Int. Mining Geology Conference, Bendigo, Victoria, Australia. Details: E-mail: conference@ausimm.com.au, Website: www.ausimm.com.au

\section{JANUARY 2004}

5-9 9th Joint MMM-Intermag Conference, Anaheim, CA, USA. Details: Courtesy Associates, Washington, USA. Tel.: 202-973-8676, Fax: 202-973-8722. E-mail: intermag@courtesyassoc.com, Website: www.intermagconference.com

\section{FEBRUARY 2004}

1-4 INFACON X - International Ferro-alloys Conference, Cape Town, South Africa. Details: E-mail: sam@saimm.co.za,website: http://www.saimm.co.za

3-4 Mineral Processing Conference, Lulea, Sweden. Details: Siv T. Berhan, Fax: +46-920-973-64, E-mail: siv.berhan@km.luth.se

19-21 International Seminar on Mineral Processing Technology 2004, Bhubaneswar, Orissa, India. Details: G.V. Rao, Fax: 0674-2581637, E-mail: gvrao@rrlbhu.res.in

23-25 SME Annual Meeting, Denver, USA. Details: SME Meetings Dept., Fax: 303-979-3461. 


\section{MARCH 2004}

22-23 Gravity Concentration '04, Perth, Australia. Details: E-mail: bwills@min-eng.com 24-26 Comminution '04, Perth, Australia. Details: E-mail: bwills@min-eng.com

\section{APRIL 2004}

18-21 VI International Conference on 'Clean Technologies for the Mining Industry', Concepcion, Chile. Details: M. Sanchez, Department of Metallurgical Engineering, University of Concepcion, , PO Box 53-C, Concepcion, Chile. Website: www. udec.cl/ctmi

19-23 World Filtration Congress, New Orleans, Louisiana, USA. Details: Wallage Leung, E-mail: wallace.leung@bakerhughes.com

\section{MAY 2004}

17-20 SWEMP 2004: 8th International Symposium on Environmental Issues and Waste Management in Energy and Mineral Production, Antalya, Turkey. Details: E-mail: terbay@atilim.edu.tr

20-24 Scientific and Clinical Applications of Magnetic Carriers, Lyon, France. Details: Dr U. Hafeli, The Cleveland Clinic Foundation, 9500 Euclid Avenue, T-28, Cleveland OH 44195, USA. E-mail: info@magneticmicrosphere.com, Website: www.magneticmicrosphere.com

\section{JUNE 2004}

7-9 International Conference 'Advances in Mineral Resource Management and Environmental Geotechnology', Chania, Crete, Greece. Details: Website: heliotopos. conference.gr/index.php?id $=670$

16-18 Reagents in Mineral Processing, Falmouth, Cornwall, UK. Details: E-mail: bwills@min-eng.com

20-24 7th International Conference on Nanostructured Materials, Wiesbaden, Germany. Details: Website: www.nano2004.org

24-26 8th Conference on Environment and Mineral Processing, Ostrava, Czech Republic. Details: P. Fecko, VSB - Technical University, Ostrava, Czech Republic. E-mail: peter. fecko@vsb.cz

\section{AUGUST 2004}

2-6 International Conference on Magnetic Fluids (ICMF10), Guaruje, State of Sao Paulo, Brazil. Details: Website: http://www.unb.br/icmf10/ 
22-25 5th International Symposium on Waste Processing and Recycling in the Mineral and Metallurgical Industries, Hamilton, Ontario, Canada. Details: M. Sudbury, Tel.: 905-339-2733, Fax: 905-339-3465, E-mail: msudbury@cogeco.ca

22-25 5th UBC-McGill Bi-Annual International Symposium on Fundamentals of Mineral Processing, Hamilton, Ontario, Canada. Details: Dr J.S. Laskowski, E-mail: js1@mining. ubc.ca

\section{SEPTEMBER 2004}

19-24 8th International Congress on Applied Mineralogy, Aguas de Lindoia, Brazil. Details: H. Kahn, E-mail: henrkahn@usp.br

26-29 REWAS 2004, Global Symposium on Recycling Waste Treatment and Clean Technology, Madrid, Spain. Details: Dr R. Solozabal, E-mail: rsoloza@inasmet.es

\section{NOVEMBER 2004}

7-11 Conference on Magnetism and Magnetic Materials, Jacksonville, FL, USA.

8-9 Solid-Liquid Separation '04, Cape Town, South Africa. Details: B. Wills, Tel.: 44-7768-234-121, Fax: 44-1326-318352, E-mail: bwills@min-eng.com

10-12 Precious Metals '04, Cape Town, South Africa. Details: B. Wills. Tel.: 44-7768-234-121, Fax: 44-1326-318352, E-mail: bwills@min-eng.com

\section{FEBRUARY 2005}

28-2 March SME Annual Meeting, Salt Lake City, USA. Details: SME Meetings Dept., Fax: 303-979-3461

\section{APRIL 2005}

International Conference on Magnet Technology MT-19, Genoa, Italy.

\section{JUNE 2005}

5-9 Centenary of Flotation Symposium, Brisbane, Australia. Details: E-mail: miriamw@ ausimm.com.au

\section{SEPTEMBER 2006}

3-8 XXIII International Mineral Processing Congress, Istanbul Turkey. Details: G. Onal, Istanbul Technical University, Mining Engineering Faculty, Istanbul, Turkey, E-mail: onalg@itu.edu.tr, Website: www.impc2006.org 